\title{
New variants in COL5A1 gene among Polish patients with Ehlers-Danlos syndrome: analysis of nine cases
}

\author{
Anna Junkiert-Czarnecka, Maria Pilarska-Deltow, Aneta Bąk, Marta Heise, Olga Haus
}

Department of Clinical Genetics, Faculty of Medicine, Collegium Medicum in Bydgoszcz, Nicolaus Copernicus University in Torun, Poland Adv Dermatol Allergol 2019; XXXVI (1): 29-33

DOI: https://doi.org/10.5114/ada.2018.79440

\begin{abstract}
Introduction: The Ehlers-Danlos syndrome (EDS) is a non-inflammatory, heritable connective tissue disorder divided into 13 types according to the 2017 International Classification of the Ehlers-Danlos syndromes. One of the subtypes of EDS, classical (cEDS), is characterized by joint hypermobility, skin hyperextensibility and atrophic scars, which are major criteria of cEDS.

Aim: In this study, the first in Central Eastern Europe, 44 patients were investigated. All of them were tested for COL5A1 mutations with direct DNA sequencing.

Material and methods: The study group included 44 patients of Polish origin, all of whom fulfilled criteria for the classical type of Ehlers-Danlos syndrome. Direct sequencing of the COL5A1, COL5A2 and COL1A1 C.934C $>$ T genes was performed for all of them. Evaluation of potential pathogenicity of detected missense mutation was conducted using SIFT (Sorting Intolerant from Tolerant), PolyPhen-2, AlignGVGD (Align Grantham Variance/Grantham Difference). The effect of the splice site mutations was predicted by Human Splicing Finder and NetGene2 tools.

Results: Among all tested patients, nine mutations of COL5A1 gene were detected (8 missense mutations and 1 splice site). The alterations identified by us are new, hitherto not described in other reports. Evaluation of the mutations by in silico tools indicate their pathogenicity.

Conclusions: Our study is the first COL5A1 gene molecular investigation conducted among cEDS patients from Central Eastern Europe. Besides new COL5A1 variant findings, we gained molecular confirmation of clinical diagnosis of cEDS. In some cases, specific and adequate evaluation and classification of EDS patients based only on clinical features, may be difficult.
\end{abstract}

Key words: Ehlers-Danlos syndrome, mutation, collagen, connective tissue, Polish population.

\section{Introduction}

The Ehlers-Danlos syndrome (EDS) (MIM \#130000) is a non-inflammatory, heritable connective tissue disorder [1-3]. According to the newest 2017 International Classification of the Ehlers-Danlos syndromes, which replaced Villefranche nosology (1997), EDS was divided into 13 types: Classical EDS (cEDS), Classical-like EDS (cIEDS), Cardiac-valvular EDS (cvEDS), Vascular EDS (vEDS), Hypermobile EDS, Arthrochalasia EDS (aEDS), Dermatosparaxis EDS (dEDS), Kyphoscoliotic EDS (kEDS), Brittle Cornea Syndrome (BCS), Spondylodysplastic EDS (spEDS), Musculocontractural EDS (mcEDS), Myopathic EDS (mEDS) and Periodontal EDS (pEDS) [4]. Villefranche clinical criteria for the classical type of EDS (cEDS) are applied in 2017 classification and include hypermobile joints, hyperextensibility of the skin and widened atrophic scars. The minor symptoms are smooth, velvety skin, molluscoid pseudotumors, easy bruising, muscle hypotonia and delayed motor development [5]. Some cEDS patients present clinical features characteristic of other subtypes, especially the hypermobile and vascular ones. Due to the variety of symptoms, classification of borderline patients by clinical examination alone is difficult [6].

The main cause of the classical type of Ehlers-Danlos syndrome is defects in type $V$ collagen. Type $V$ collagen is a quantitatively minor fibrillar collagen with wide tissue distribution. Together with type I collagen it plays the main role in collagen fibrillogenesis [7]. The most common isoform of type $V$ collagen is a heterotrimer composed of two $\alpha 1$ propeptide chains and one $\alpha 2$-propeptide chain encoded by COL5A1 and COL5A2, respectively. $\alpha 1$-propeptide chain is composed of three domains: $N$-propeptide, $\boldsymbol{\alpha}$-helix and C-propeptide [8].

In previous CEDS studies, many various mutations in COL5A1, rarely COL5A2 and mutation C.934C $>T$ in COL1A1

Address for correspondence: Anna Junkiert-Czarnecka MSc, Department of Clinical Genetics, Faculty of Medicine, Collegium Medicum, Nicolaus Copernicus University, 9 M. Curie-Skłodowskiej St, 85-094 Bydgoszcz, Poland, phone: +48 5258535 67, fax: +48 525853568 , e-mail: ajczarnecka@cm.umk.pl

Received: 11.09 .2018 , accepted: 3.10 .2018 
genes were reported [1, 6, 9-12]. The most frequent mutations in COL5A1 gene were nonsense or frameshift mutations localized mainly in $\alpha$-helix and C-propeptide domain. So far, only a few mutations localized in the highly conserved N-propeptide domain, which plays a significant role in the regulation of collagen fibril diameter, were described $[2,10,13,14]$.

\section{Material and methods}

The study group included 44 patients from 28 families of Polish origin, 31 women and 13 men, aged 3-59 years (median: 18.5). All patients fulfilled criteria for the classical type of Ehlers-Danlos syndrome. The enrolment of patients was conducted on the basis of International Classification 2017 diagnostic criteria of cEDS by experienced clinical geneticists. Joints hypermobility was evaluated on the Beighton scale. The control group consisted of 100 volunteers from the general Polish population. All EDS patients and control group participants provided informed consent. The study was also approved by the Ethics Committee of the Collegium Medicum in Bydgoszcz, Nicolaus Copernicus University in Torun.

Direct sequencing of the COL5A1, COL5A2 and exon 14 (mutation c.934C >T) COL1A1 gene was performed for all participants. Genomic DNA (gDNA) was extracted from leukocytes by QIAamp DNA Mini Kit (Qiagen, Germany) using standard procedures. Primers were designed using Primer3 (http://bioinfo.ut.ee/primer3-0.4.0/) tool. Exons and intronflanking regions of all tested genes were amplified by PCR (PCR profiles, primers sequence available upon request). Sequencing reaction was conducted on PCR product with BigDye Terminator v3.1 Cycle Sequencing Kit (Applied Biosystems) according to the manufacturer's instructions. The sequences were analyzed using ABI PRISM 3130 (Applied Biosystems). Evaluation of potential pathogenicity of detected missense mutation was done using SIFT (Sorting Intolerant from Tolerant) [15], PolyPhen-2 [10] and AlignGVGD (Align Grantham Variance/Grantham Difference) [16]. The effect of the splice site mutations was predicted by Human Splicing Finder and NetGene2 tools. Mutational analysis was performed on NCBI reference sequences: NM_000093.4, NM_000393.4, NM_000088.3. All investigations were performed in two independent blood probes from every patient.

\section{Results}

\section{Variants in the COL5A2 and mutation c.934C $>T$ in COL1A1 genes}

In the COL5A2 gene, pathogenic variants were not detected, as was the case in the investigated group mutation c.934C $>\mathrm{T}$ in COL1A1.

\section{Variants in COL5A1 gene}

In 4 (9.09\%) out of 44 cEDS patients, common alteration (previously described) c.1588G >A (p.G530S) in exon
13 was detected $[17,18]$. In the general Polish population, the frequency of this variant was assessed as $4 \%$ (data unpublished). Presence of c.1588G >A alteration did not exclude patients from further investigation.

In 9 patients ( 5 children and 4 adults; 6 females and 3 males) from 44, new mutations were detected. All these patients were not carriers of c.1588G $>$ A variant. The clinical data are presented in Table 1. A family history of cEDS was presented in 7 of 9 patients. Joint hypermobility was present in all 9 patients, however, the tendency for joint dislocations was present only in 5 . Seven out of 9 patients complained of skin hyperextensibility and various degrees of easy bruising. Slow wound healing was present in $1 \mathrm{pa}-$ tient. Additional clinical manifestations included: muscle hypotonia, delayed motor development, hip dysplasia, flat feet, knees and heel valgity, scoliosis, short stature, osteoporosis, refluxes, diarrheas, navel hernia, mitral valve prolapse, high palate, blue sclera, chronic joints pain, whole body pain and chronic fatigue. There were no differences in symptoms intensity between children and adults and between men and women.

\section{Molecular analysis}

Molecular analysis was carried out on the coding parts (with parts of introns from 5' and 3' end of all tested exons) of the COL5A1 gene. 9 mutations identified in this study were: 8 missense mutations and one splice site mutation. They affected different domains of the $\alpha 1(\mathrm{~V})$ chain. Pathogenicity of identified mutations was evaluated using SIFT, PolyPhen-2, AlignGVGD for missense mutations and Human Splicing Finder, NetGene2 for splice site mutation. The relevant data are shown in Table 2.

\section{Missense mutations}

All found 8 missense mutations (c.193C > T, c.514G >T, c.367C > G, c.944C > T, c.1089C > G, c.3418G >A, c.2588A > T, c. $4483 \mathrm{G}>\mathrm{A}$ ) were evaluated using bioinformatic tools (Table 2). Mutations c.193C > T, c.514G > T, c.367C > G, c.944C > T and $c .1089 \mathrm{C}>\mathrm{G}$ were located in the N-terminal part of the protein. Only for variants c.193C > T and c.514C>T (patient 011) every used tool indicated a deleterious character. For the rest of detected variants (c.367C $>$ G, c.944C $>$ T and c.1089C > G), ambiguous results were obtained.

Mutations c.3418G >A, c.2588A>T and c.4483G >A are located in the $\alpha$-helix domain. Variant c.3418G >A was present in patient 089 and her sister (089S). Evaluation was unambiguous also in this case. PolyPhen-2 showed it is a pathogenic variant, when SIFT and AlignGVGD showed it is not deleterious. In patient 089, a second COL5A1 c.2588A>T (p.E863V) change was also detected. It was recorded in the NCBI SNP base (www.ncbi.nlm.nih.gov/snp) and in ClinVar base (www.ncbi.nlm.nih.gov/clinvar). Minor allele frequency according to 1000 Genomes Project was $T=0.4 \%$, clinical significance was classified as "uncertain significance". In silico prediction indicated the pathogenic character of this 
Table 1. Clinical findings of 9 patients with new mutations

\begin{tabular}{|c|c|c|c|}
\hline $\begin{array}{l}\text { Patient laboratory } \\
\text { number }\end{array}$ & Sex/age & Clinical features & $\begin{array}{l}\text { Family history } \\
\text { of EDS }\end{array}$ \\
\hline 133 & $M / 15$ & $\begin{array}{l}\text { Joints hypermobility and pain, flat feet, scoliosis, chronic osteoporosis, short } \\
\text { stature, muscle hypotonia, diarrheas, chronic fatigue syndrome }\end{array}$ & + \\
\hline 083 & $M / 7$ & $\begin{array}{l}\text { Joints hypermobility, knees and heels valgity, scoliosis, muscle hypotonia, delayed } \\
\text { motor development, high palate, hyperextensibility of the skin, velvety skin, easy } \\
\text { bruising, navel hernia, reflux, blue sclera }\end{array}$ & + \\
\hline 011 & $M / 16$ & $\begin{array}{l}\text { Joints hypermobility and dislocations, feet valgity, osteoporosis, high palate, } \\
\text { hyperextensibility of the skin, whole body pain }\end{array}$ & - \\
\hline 182 & $\mathrm{~F} / 20$ & $\begin{array}{l}\text { Joints hypermobility and dislocations, feet and knees valgity, high palate, easy } \\
\text { bruising, slow wound healing, chronic fatigue syndrome }\end{array}$ & - \\
\hline 105 & $\begin{array}{l}\mathrm{F} / 29 \text { (mother } \\
\text { of 106) }\end{array}$ & $\begin{array}{l}\text { Joints hypermobility and dislocations, flat feet, hyperextensibility of the skin, easy } \\
\text { bruising }\end{array}$ & + \\
\hline 106 & $\begin{array}{l}\text { F/7 (daughter } \\
\text { of 105) }\end{array}$ & $\begin{array}{l}\text { Joints hypermobility, congenital hip dysplasia, scoliosis, hyperextensibility of the } \\
\text { skin }\end{array}$ & + \\
\hline 089 & $\begin{array}{l}\text { F/62 (sister } \\
\text { of 089S) }\end{array}$ & $\begin{array}{l}\text { Joints hypermobility and dislocations, congenital hip dysplasia, flat feet and feet } \\
\text { valgus, scoliosis, delayed motor development, osteoporosis, hyperextensibility } \\
\text { of the skin, easy bruising, velvety skin, arrhythmia, mitral insufficiency, } \\
\text { gastrointestinal disorders }\end{array}$ & + \\
\hline 0895 & $\begin{array}{l}\text { F/63 (sister } \\
\text { of 089) }\end{array}$ & Joints hypermobility and dislocations, velvety skin & + \\
\hline 075 & $F / 12$ & $\begin{array}{l}\text { Joints hypermobility, gothic palate, scoliosis, smooth, velvety skin, mitral valve } \\
\text { prolapse, generalized pain }\end{array}$ & + \\
\hline
\end{tabular}

Table 2. In silico analysis of new mutations found in Polish patients with Ehlers-Danlos syndrome.

\begin{tabular}{|c|c|c|c|c|c|c|c|c|}
\hline Patient & Mutation & $\begin{array}{l}\text { Amino acid } \\
\text { change }\end{array}$ & $\begin{array}{l}\text { Exon/ } \\
\text { intron }\end{array}$ & SIFT & $\begin{array}{l}\text { Align } \\
\text { GV GD }\end{array}$ & PolyPhen & HSF & Net Gene2 \\
\hline 011 & c. $193 C>T$ & p.R65W & ex2 & Deleterious & $\mathrm{C} 65$ & $P$ & $\mathrm{Nt}$ & $\mathrm{Nt}$ \\
\hline 011 & c. $514 \mathrm{G}>\mathrm{T}$ & p.V172F & ex4 & Deleterious & C45 & $P$ & $\mathrm{Nt}$ & $\mathrm{Nt}$ \\
\hline 182 & c. $367 C>G$ & p.Q123E & ex3 & Deleterious & $\mathrm{C} 25$ & $P$ & $\mathrm{Nt}$ & $\mathrm{Nt}$ \\
\hline 133 & c. $944 C>T$ & р.Т315M & ex7 & Tolerated & $\mathrm{C} 65$ & $P$ & $\mathrm{Nt}$ & $\mathrm{Nt}$ \\
\hline 105 & c. $1089 C>G$ & p.N363K & ex7 & Tolerated & $\mathrm{C} 65$ & B & $\mathrm{Nt}$ & $\mathrm{Nt}$ \\
\hline 106 & c. $1089 C>G$ & p.N363K & ex7 & Tolerated & $\mathrm{C} 65$ & B & $\mathrm{Nt}$ & $\mathrm{Nt}$ \\
\hline 089 & c. $3418 \mathrm{G}>\mathrm{A}$ & p.V1138M & ex43 & Tolerated & $\mathrm{C} 15$ & $P$ & $\mathrm{Nt}$ & $\mathrm{Nt}$ \\
\hline 089 & c. $2588 \mathrm{~A}>\mathrm{T}$ & p.E863V & ex30 & Deleterious & $\mathrm{C} 65$ & $\mathrm{P}$ & $\mathrm{Nt}$ & $\mathrm{Nt}$ \\
\hline 0895 & c. $3418 \mathrm{G}>\mathrm{A}$ & p.V1138M & ex43 & Tolerated & $\mathrm{C} 15$ & $P$ & $\mathrm{Nt}$ & $\mathrm{Nt}$ \\
\hline 083 & c. $4483 G>A$ & p.G1495S & ex58 & Deleterious & C55 & $P$ & $\mathrm{Nt}$ & $\mathrm{Nt}$ \\
\hline 075 & c. $1662+9 \mathrm{~T}>\mathrm{C}$ & - & int13 & $\mathrm{Nt}$ & $\mathrm{Nt}$ & $\mathrm{Nt}$ & $\mathrm{N}$ & $P$ \\
\hline
\end{tabular}

Nt - not tested, P-pathogenic, B - benign, N-neutral, AlignGVGD range: C65-most likely pathogenic-CO-less likely pathogenic.

substitution. Comparing clinical findings of the two sisters, we can note that patient 089, who is a carrier of two variants, has got a much harder clinical picture than her sister who is a carrier of only one COLSA1 variant.

Another mutation detected in cEDS patient (laboratory number 083) was c.4483G $>$ A mutation. The arguments in favor of the pathogenicity of c.4483G >A mutation were its localization in $\alpha$-helix domain and bioinformatic analysis. All used tools indicated this variant's pathogenicity.

\section{Splice site mutation}

The only splice site mutation found was c.1662+9T>C in intron 13 (patient 075). NetGene2 tools used to assess the effect of this substitution indicated that it alters an 
exonic ESE site and may affect RNA splicing but Human Splicing Finder evaluated this alteration as neutral.

For all detected variants (missense and splice site), 100 healthy persons from the general Polish population control group were tested and they were not carriers of any described alteration in this manuscript.

\section{Discussion}

The investigations described in the article were the first for cEDS patients from Central Eastern Europe. Patients were evaluated and classified according to the 2017 International Classification of the Ehlers-Danlos syndromes. Similarly to patients described by Malfait et al., Ritelli et al. and Symoens et al. Polish patients presented variability of cEDS phenotype $[6,11,18]$. Skin involvement (hyperextensibility of skin and/or smooth, velvety skin, atrophic scars) and hypermobility of joints were dominant phenotypic conditions. These data show that cEDS patients share some clinical features with the hypermobility type of EDS and that certain diagnosis of cEDS in our patients could be established mainly based on mutations in COL5A1 gene. Determining a type of EDS based only on the clinical examination may be difficult in many cases. Basing on identified cEDS phenotypic features of close relatives of patients, Ritelli et al. reclassified as cEDS 10 from 40 borderline classical-hypermobile patients, who did not fulfil three major phenotypic criteria of cEDS. Clinical diagnosis of cEDS was confirmed by COL5A1/COL5A2 molecular test [2].

Variants described in this paper are new, hitherto not detected in other COL5A1 investigations [6, 11]. All but one were of missense type, with the remaining one being a splice site variant. There were no nonsense, frame shift mutations, like those which were discovered among Italian or Belgian cEDS patients $[6,11,18]$. The mutations described by others were localized in all COL5A1 domains, mainly in $\alpha$-helix and C-propeptide domain and rarely in highly conservative N-propeptide $[6,11,18]$. In the Polish cEDS patients we analyzed, mutations were localized only in $\mathrm{N}$-propeptide and $\boldsymbol{\alpha}$-helix domain. No mutation in C-propeptide domain has been detected until now.

In silico analysis tools were used to evaluate possible pathogenicity of found mutations. This approach to estimate impact of mutations on type $V$ collagen structure and function and subsequently on patients' condition was proposed by Symoens et al., Malfait et al. and Ritelli et al. [6, 11, 18]. Variants C.193C >T, C.514G $>$ T and c.4483G $>$ A were clearly evaluated by all investigation tools used as deleterious. Assessment of other variants was ambiguous but its impact on patients' condition cannot be excluded. Missense mutations described up to now in N-propeptide and $\alpha$-helix domain of COL5A1 were evaluated as damaging conformation and function of type $V$ collagen. Mutations in this part of the gene, described previously by other authors, disrupted interac- tions between type $V$ collagen and extracellular matrix (ECM) components and interfered with the formation of type $V$ collagen molecule $[3,6,8,11,13,19]$. Intronic mutation was indicated as pathogenic by NetGene- 2 tool but as neutral by the Human Splicing Finder. Certain definition of this variant is possible after further investigations such as RNA analysis.

Phenotypic results of a large variety of mutations located along the entire COL5A1 gene and a large number of new, still unclassified mutations in cEDS may be difficult to interpret and specify. Evaluation of variants in silico despite some limitations may allow for estimation of their phenotypic results (deleterious or not) and for adequate genetic counseling.

\section{Acknowledgments}

This investigation was supported by Nicolaus Copernicus University statutory research number 286.

We thank all patients and their families for participating in this study.

\section{Conflict of interest}

The authors declare no conflict of interest.

\section{References}

1. Malfait F, De Paepe A. Molecular genetics in classic EhlersDanlos syndrome. Am J Med Genet 2005; 139C: 17-23.

2. Malfait F, Wenstrup R, De Paepe A. Clinical and genetic aspects of Ehlers-Danlos syndrome, classic type. Genet Med 2010; 12: 597-605.

3. Michalickova K, Susic M, Willing MC, et al. Mutations of the alpha2(V) chain of type $V$ collagen impair matrix assembly and produce Ehlers-Danlos syndrome type I. Hum Mol Genet 1998; 7: 249-55.

4. Malfait F, Francomano C, Byers P, et al. The 2017 international classification of the Ehlers-Danlos syndromes. Am J Med Genet C Semin Med Genet 2017; 175: 8-26.

5. Beighton P, De Paepe A, Steinmann B, et al. Ehlers-Danlos syndromes: revised nosology, Villefranche 1997. Am J Med Genet 1998; 77: 31-7.

6. Symoens S, Syx D, Malfait F, et al. Comprehensive molecular analysis demonstrates type $V$ collagen mutations in over $90 \%$ of patients with classic EDS and allows to refine diagnostic criteria. Hum Mutat 2010; 10: 1485-93.

7. Birk DE. Type $V$ collagen: heterotypic type I/V collagen interactions in the regulation of fibril assembly. Micron 2001; 32: 223-37.

8. Takahara K, Hoffman GG, Greenspan DS. Complete structural organization of the human alpha $1(\mathrm{~V})$ collagen gene (COL5A1): divergence from the conserved organization of other characterized fibrillar collagen genes. Genomics 1995; 29: 588-97.

9. Mitchell AL, Schwarze U, Jennings JF, Byers PH. Molecular mechanisms of classical Ehlers-Danlos syndrome (EDS). Hum Mutat 2009; 30: 995-1002.

10. Paladin L, Tosatto SC, Minervini G. Structural in silico dissection of the collagen $\mathrm{V}$ interactome to identify genotype- 
phenotype correlations in classic Ehlers-Danlos syndrome (EDS). FEBS Lett 2005; 589: 3871-8.

11. Ritelli M, Dordoni C, Venturini M, et al. Clinical and molecular characterization of 40 patients with classic Ehlers-Danlos syndrome: identification of 18 COL5A1 and 2 COL5A2 novel mutations. Orphanet J Rare Dis 2013; 8: 58.

12. Takahara K, Schwarze U, Imamura Y, et al. Order of intron removal influences multiple splice outcomes, including a twoexon skip, in a COL5A1 acceptor-site mutation that results in abnormal pro-alpha1(V) N-propeptides and Ehlers-Danlos syndrome type I. Am J Hum Genet 2002; 71: 451-65.

13. Symoens S, Malfait F, Vlummens P, et al. A novel splice variant in the N-propeptide of COL5A1 causes an EDS phenotype with severe kyphoscoliosis and eye involvement. PLoS One 2011; 6: e20121.

14. Wenstrup R, Florer J, Brunskill E, et al. Type V collagen controls the initiation of collagen fibril assembly. J Biol Chem 2004; 279: 53331-37.

15. Kumar P, Henikoff S, Ng PC. Predicting the effects of coding non-synonymous variants on protein function using the SIFT algorithm. Nat Protoc 2009; 4: 1073-81.

16. Tavtigian SV, Deffenbaugh AM, Yin L, et al. Comprehensive statistical study of 452 BRCA1 missense substitutions with classification of eight recurrent substitutions as neutral. J Med Genet 2006; 43: 295-305.

17. Giunta C, Nuytinck L, Raghunath M, et al. Homozygous Gly530Ser substitution in COL5A1 causes mild classical Ehlers-Danlos syndrome. Am J Med Genet 2002; 109: 28490.

18. Malfait F, Coucke P, Symoens S, et al. The molecular basis of classic Ehlers-Danlos syndrome: a comprehensive study of biochemical and molecular findings in 48 unrelated patients. Hum Mutat 2005; 25: 28-37.

19. Symoens S, Renard M, Bonod-Bidaud C, et al. Identification of binding partners interacting with the alpha1-N-propeptide of type V collagen. Biochem J 2011; 433: 371-81. 